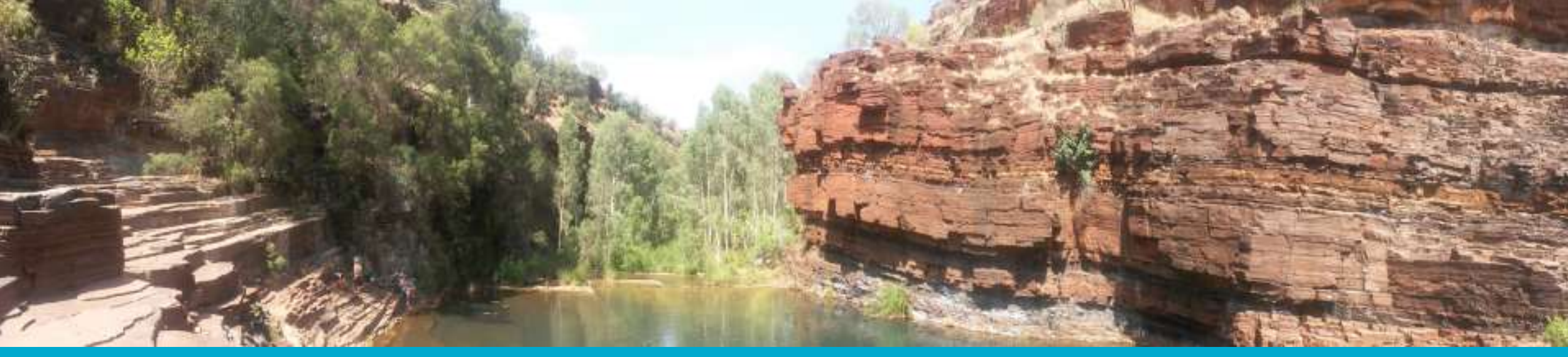

\title{
Implementing the International Geo Sample Number (IGSN) in Australia
}

Jens Klump, Anusuriya Devaraju, Lesley Wyborn, Irina Bastrakova, Pavel Golodoniuc, Brent McInnes, Simon Cox

24 October 2017 | GSA 2017

\section{MINERAL RESOURCES}

www.csiro.au

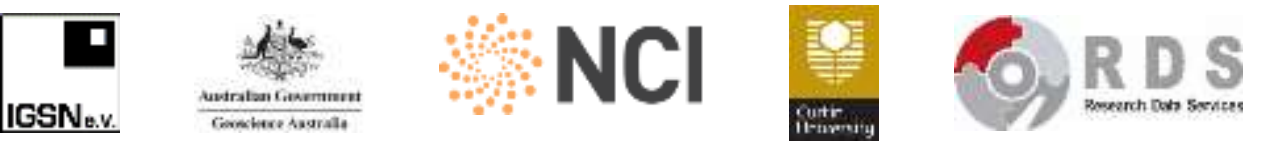




\section{What is IGSN and what does it do?}

- Provides identifiers that are guaranteed to be unique via a hierarchical governance system (like assigning IP addresses)

- Facilitates internet-based discovery and access to physical samples:

- Web applications and programmatic access to sample metadata catalogues

- Networks with sample repositories and data centres

- Ensures preservation of, and access to sample data

- Aids in the identification of samples in the literature and of data derived from them

- Try it out: http://igsn.org/ICDP5054ESYI201 or http://igsn.org/AU1101 


\section{IGSN in Australia}

- IGSN currently has three members in Australia:

- CSIRO

- Geoscience Australia

- Curtin University

- Joint governance of the use of IGSN in Australia.

- Community building (e.g. workshops) to introduce sample identifiers to other institutions and other science disciplines.

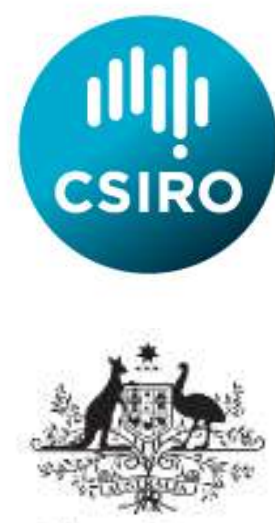

Australian Government

Geoscience Australia

Curtin

University 


\section{IGSN @ CSIRO}

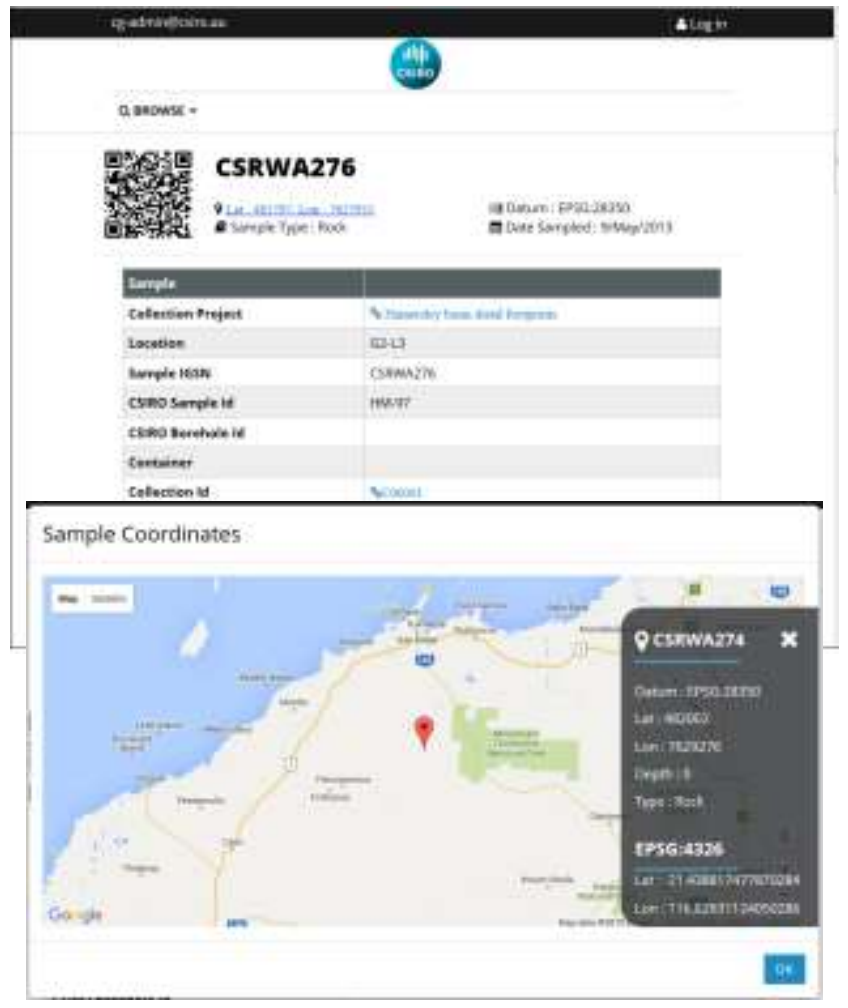

- CSIRO became a member of IGSN in 2013.

- IGSN are currently used for:

- Collection of the Australian Resources Research Centre

- Mineral Reflectance Spectra Reference Collection

- Capricorn Distal Footprints minerals exploration project

- Future use cases:

- Soils collection

- Insect collection 


\section{Tracking the sample life cycle}

IGSN is used in CSIRO for tracking samples and to support sample logistics.

- In the field: unambiguous identification, metadata capture with mobile app.

- In the lab: identification and tying data to samples.

- Storage: identify collections and samples in storage, catalogue, manage sample logistics.

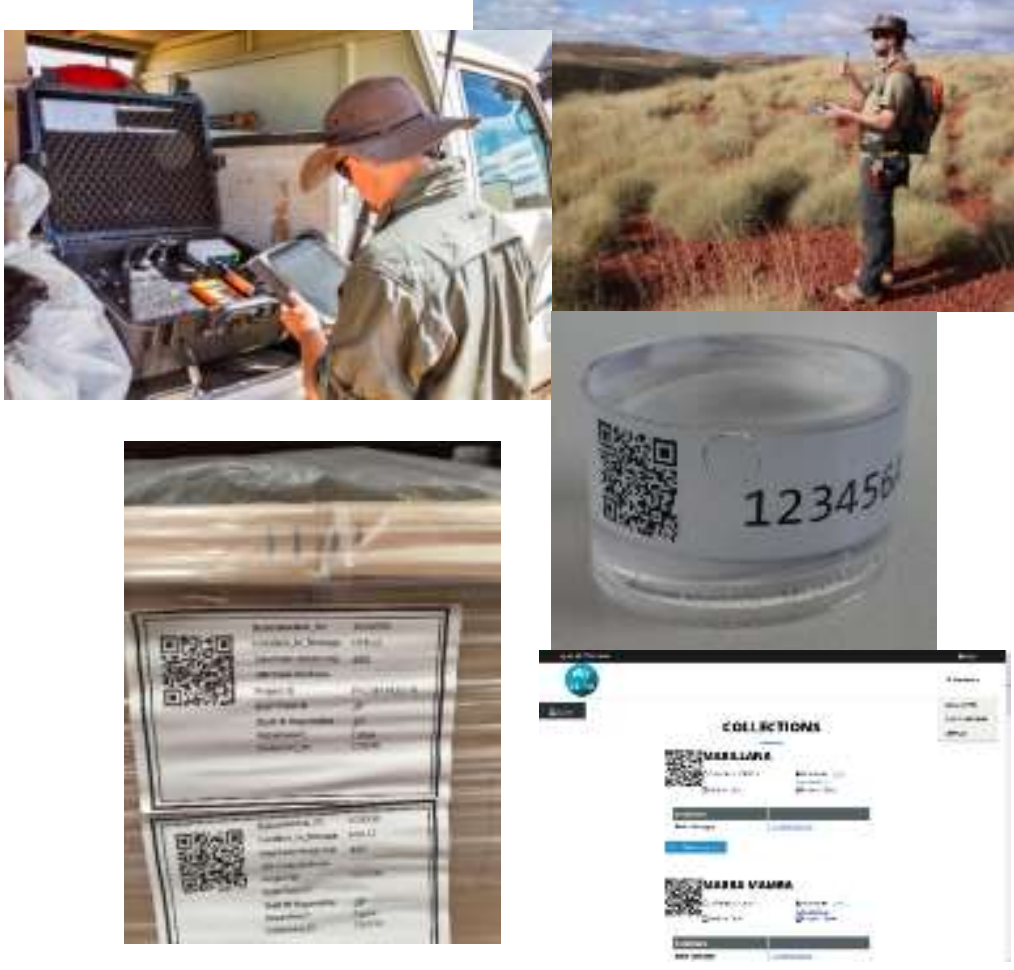




\section{Linking Samples with Data and Publications}

Specimen (Rock Store)

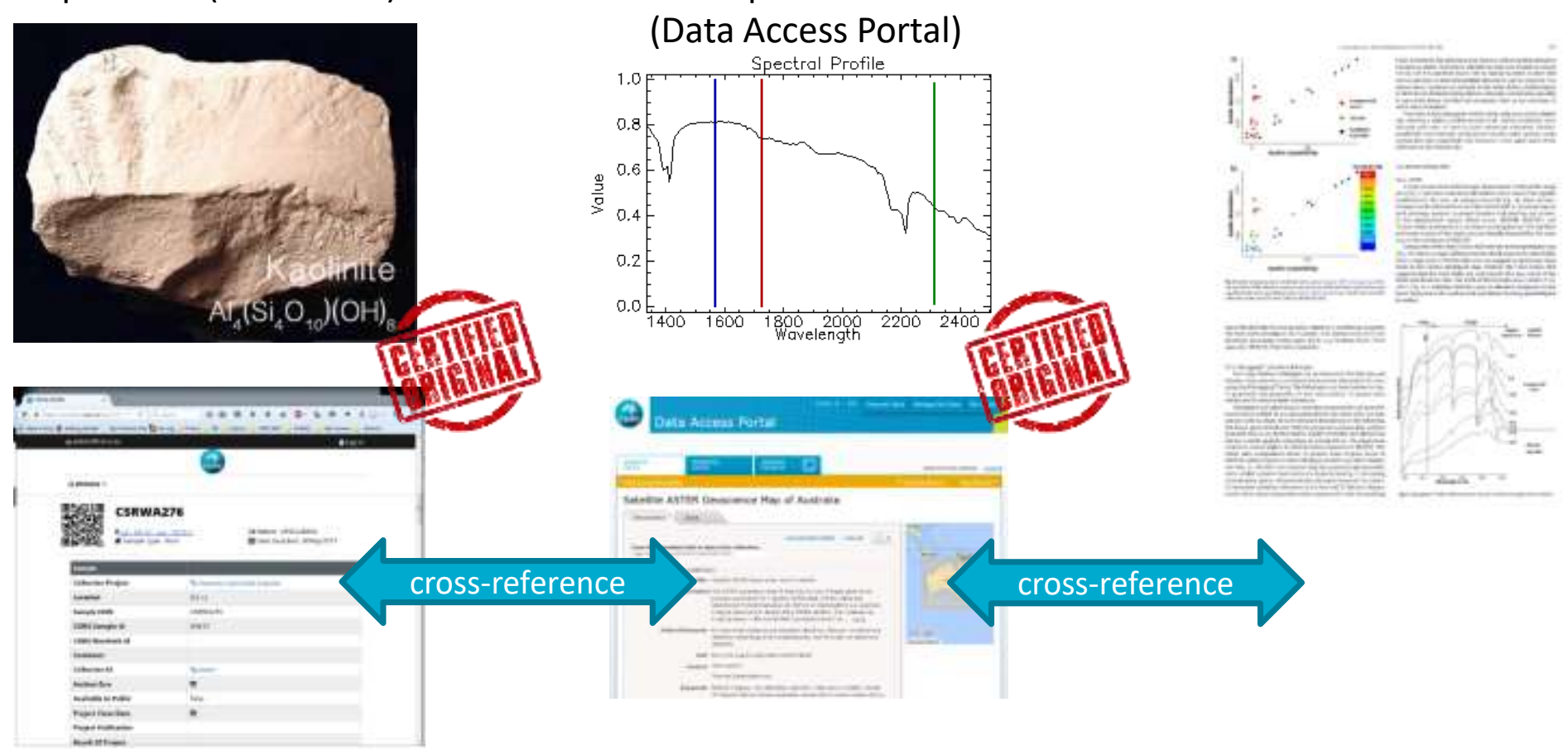




\section{IGSN@Geoscience Australia}

Applyng gecscience to Australi's most important chalenges

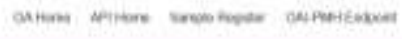

Sample

IGSN Ontology view

$-1=$

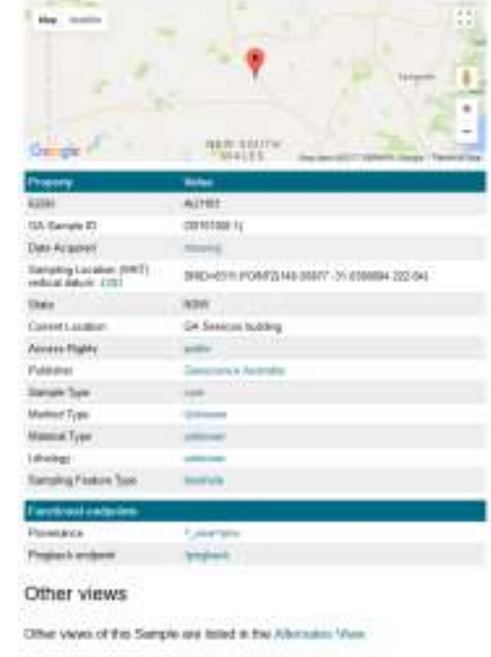

Citation
- GA became a member of IGSN in 2014.

- IGSN are currently used in the GA collection.

- 1.6 Million samples registered.

- GA is the IGSN Registration Agent for the geological surveys of the Australian states and territories. 


\section{IGSN@Curtin}

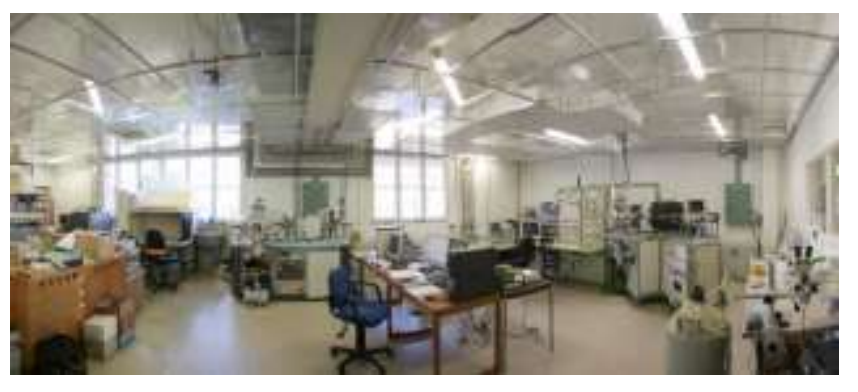

- Curtin University became a member of IGSN in 2015.

- IGSN is currently used in the John de Laeter Centre for Geochemistry.

- IGSN and data management are supported by the Curtin

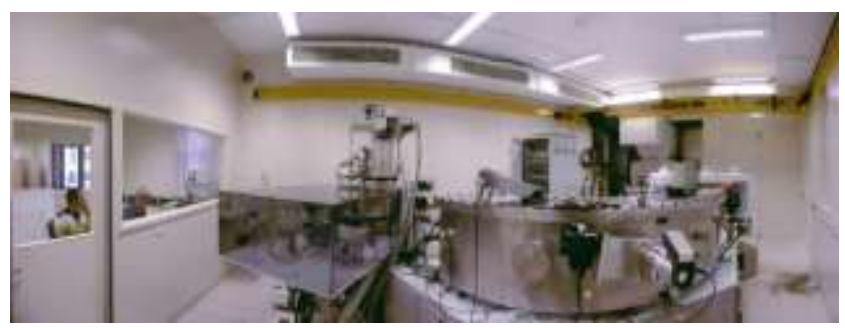
University Library.

- JdLC, CSIRO, Univ. Western Australia and Geol. Survey of Western Australia work together in the Natural Resources Research Precinct. 


\section{Governance Model}

- The governance model of IGSN is based on hierarchical delegation.

- IGSN identifiers are registered through IGSN agents.

- Each IGSN agent is given namespaces for the registration of IGSN.

- Examples:

- AU... Geoscience Australia

- CS... CSIRO

- CSCAP... CDF Project (CSIRO) 


\section{Australian IGSN Portal}

- A grant from the NCRIS Research Data Services programme made it possible to develop a demonstrator for a common Australia Geo Sample Portal.

- Metadata are harvested into a common metadata portal to facilitate the discovery of samples curated by Australian IGSN members.

- Samples are described in a common metadata schema based on the international IGSN description schema.

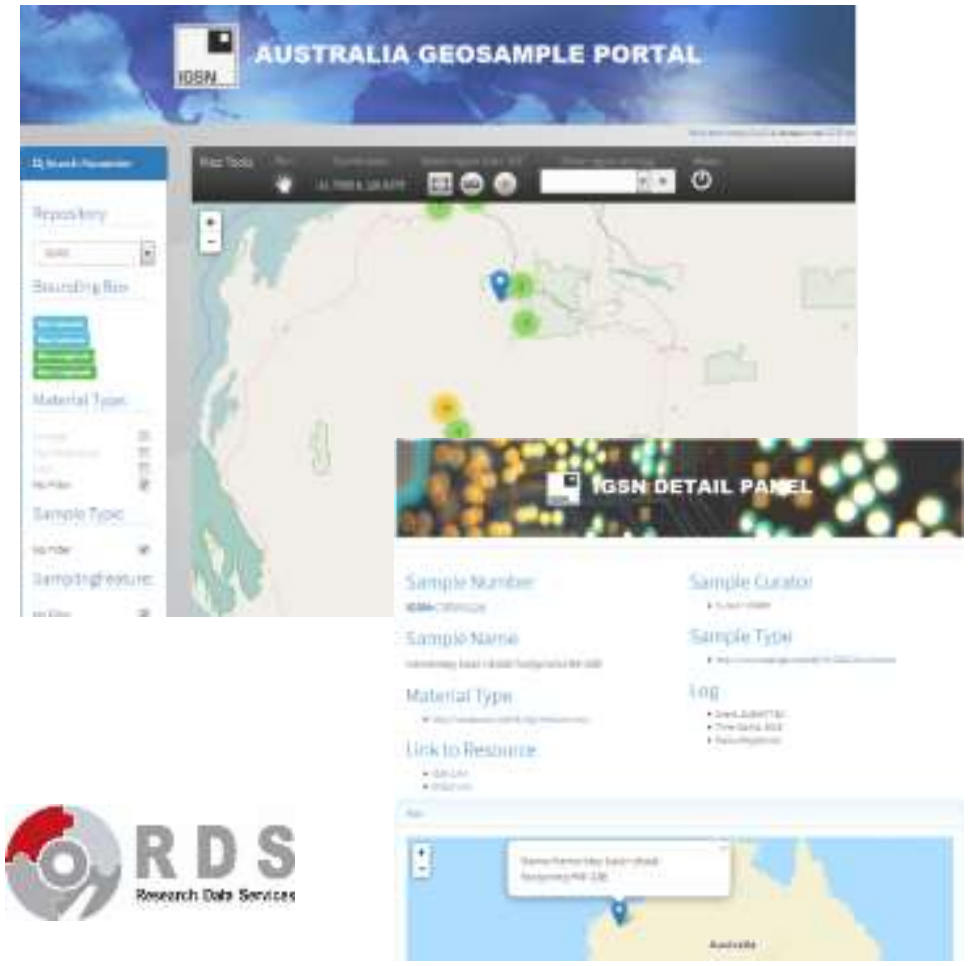




\section{Technical Base}

- IGSN builds upon an existing technical base and community.

- IGSN are based on the Handle System.

- The IGSN technical architecture is developed in close alignment with DataCite.

- http://igsn.github.io (documentation)

- https://github.com/igsn (repository)

○

International Geo Sample Number IGSN e.V.

IGSN

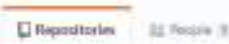

We

metadat.

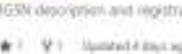

igsn.githubsio

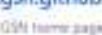

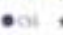

public

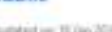

oaip 


\section{Samples Moving Between Institutions}

-What happens when samples move from one institution to another?

- Case 1: Laboratory

- IGSN available: The laboratory uses the already assigned IGSN.

- IGSN not available: The laboratory assigns a new IGSN.

- Case 2: Subsampling

- A subsample should be identified by its own IGSN. This case depends on the details of the setting.
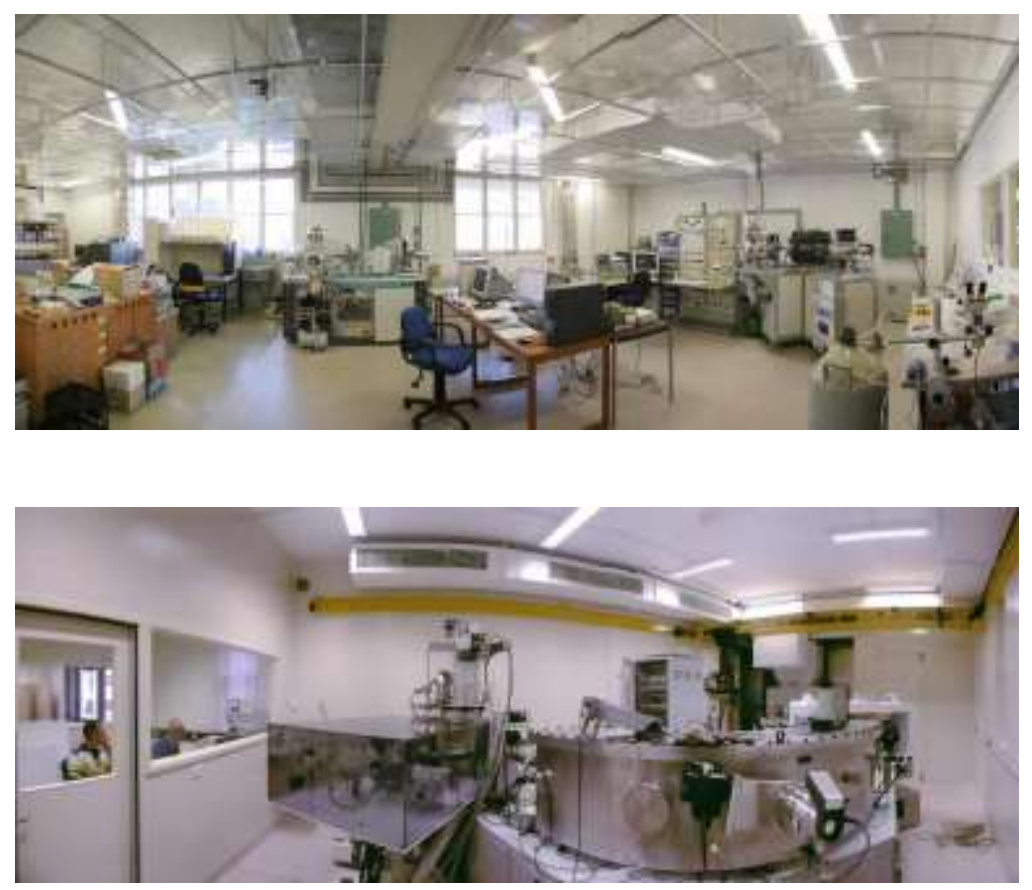


\section{Future Outlook}

- Build a developer community around IGSN, document best practices, build reference implementations of services.

- Expand identifying and linking to objects in other domains.

- Other domains start reusing IGSN technology.

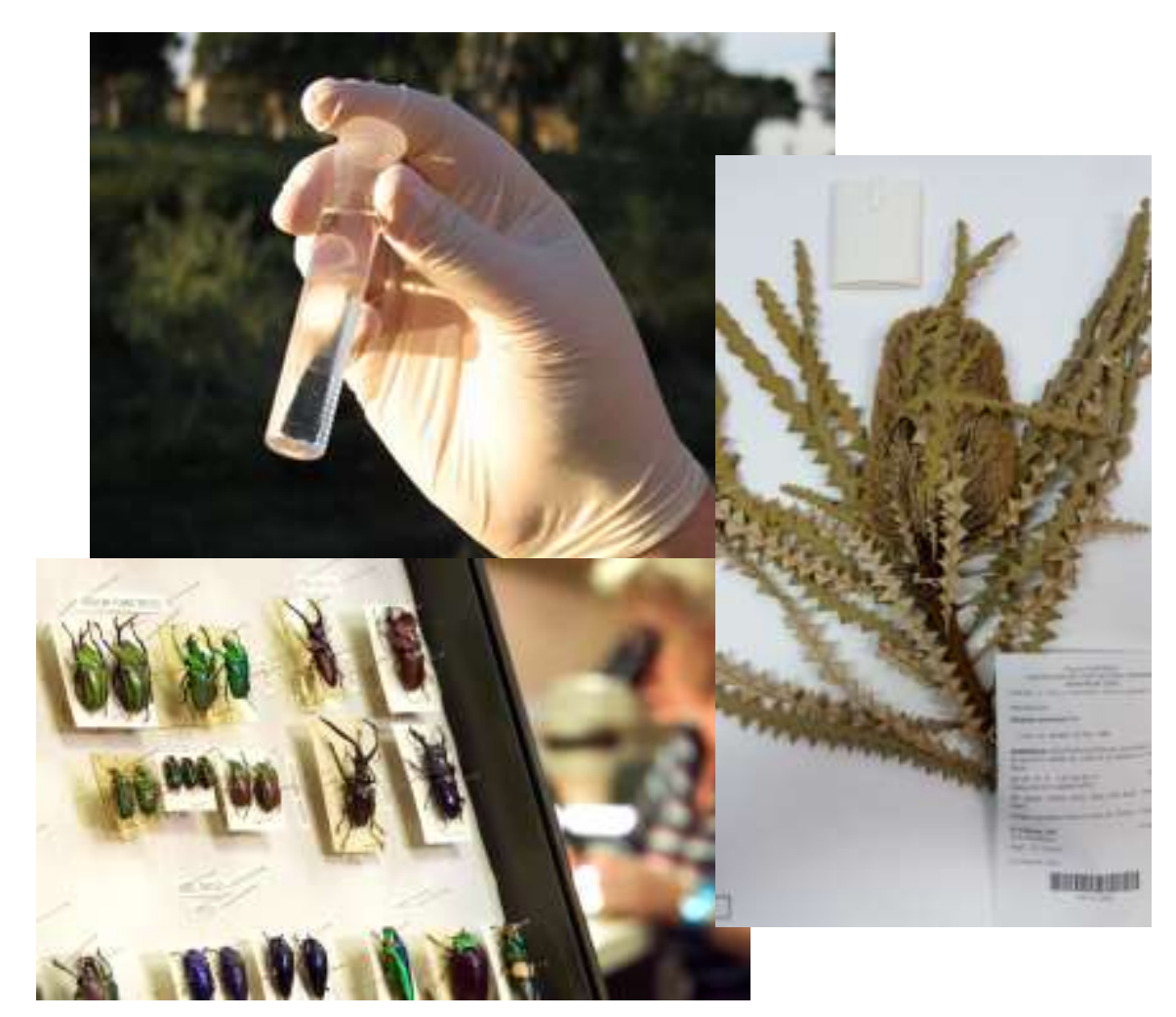




\section{Conclusions}

- IGSN was introduced in Australia through a collaboration of national labs (CSIRO), government agencies (GA) and academia (Curtin Uni).

- CSIRO, GA and Curtin Uni work together to build a community of IGSN users, develop common sample descriptions and online catalogues.

- The use of IGSN is to be expanded to novel use cases.

- General info:

- Technical info:

- Demo portal: http://www.igsn.org http://igsn.github.io http://igsn.org.au 
